\title{
Segmentación y densificación del proceso de fragmentación residencial en la Ciudad de Córdoba, Argentina (1991-2010)
}

Martín Lemma. Consejo Nacional de Investigaciones Científicas y Técnicas (CONIСET), Buenos Aires, Argentina.

RESUMEN | A partir del análisis de las estrategias de localización, segmentación y densificación de los barrios cerrados en la Ciudad de Córdoba entre 1991 y 2010, este artículo estudia la evolución del proceso de fragmentación urbana y residencial de la ciudad en el marco de la globalización. Durante estas dos décadas, se verifica el avance de los barrios cerrados como espacio para el hábitat de los grupos pertenecientes a la cúpula socioeconómica cordobesa. Más aún, se diversifica el mercado residencial y aumenta la densidad bruta de las cuatro tipologías de barrios cerrados construidas.

PALABRAS CLAVE | barrios cerrados, fragmentación urbana, globalización.

ABSTRACT | Based on the analysis of the strategies of location, segmentation and densification of gated communities in the City of Córdoba between 1991 and 2010, this article studies the evolution of the urban and residential fragmentation process of the city in the framework of globalization. During these two decades, it is possible to verify the advance of gated communities as a space for the habitat of Cordoba's leading socioeconomic groups. Results show that the residential market has diversified and the gross density of the four types of gated communities has increased.

KEYWORDS | gated communities, urban fragmentation, globalization. 


\section{Introducción}

En Latinoamérica, el espacio urbano es parte del escenario donde se manifiestan diversas transformaciones que ilustran un particular momento de cambios impulsados por la globalización. Es por esa vía que las ciudades expresan el viraje de los países de la región hacia un modelo globalizado de la economía y, junto con ello, la consolidación de políticas de desarrollo neoliberal (De Mattos, 2002).

A pesar de que el crecimiento de las ciudades está vinculado estrechamente al desarrollo de diferentes funciones urbanas — como el comercio, la industria, el servicio, entre otras - el uso residencial es el que demanda un mayor consumo de suelo y, consecuentemente, la vivienda es el negocio sobre el cual se despliegan más intensivamente las estrategias de las empresas de desarrollo urbano (Capel, 2002).

Diferentes trabajos académicos y de gestión gubernamental han alertado sobre las problemáticas derivadas del proceso de expansión de la mancha urbana de la Ciudad de Córdoba (Debat, 2016; Díaz Terreno, 2011; Irós, 1991; Marengo, 2013; Municipalidad de la Ciudad de Córdoba, 1996, 2001, 2005). ${ }^{1}$ Algunas de las cuestiones recurrentes que aparecen en estos trabajos son los altos costos económicos de la infraestructura pública necesaria para dar respuesta a una mancha urbana extendida, la falta de planificación respecto de qué sectores son prioritarios para una expansión, y la urbanización de suelo productivo o —incluso-incompatible con otras funciones colindantes.

En los documentos de planificación, la expansión de la mancha urbana es uno de los pocos problemas sobre los que las diversas gestiones han logrado establecer un consenso (Lemma, 2017). En concreto, entre 1991 y 2010, el porcentaje de superficie urbanizada o por urbanizar (respecto de la superficie total del municipio) sufrió un incremento desde el 33,25\% al 41,28\%; mientras tanto, la tasa de crecimiento poblacional continúa decreciendo desde 1970 (Municipalidad de la Ciudad de Córdoba, 2012). En este sentido, se determina que el crecimiento de la mancha urbana no responde al incremento poblacional, sino a la introducción de otras formas de organización de esa población en el espacio urbano.

En Argentina, la producción de la vivienda es llevada a cabo por una multiplicidad de actores, entre los que predominan las empresas privadas de desarrollo urbano, el Estado (en sus diferentes jerarquías), las cooperativas de vivienda (en Córdoba, la más importante de ellas es Cooperativa Horizonte), las organizaciones no gubernamentales (como TECHO), diversos movimientos sociales y los individuos. En la Ciudad de Córdoba, el sector privado es el principal promotor de barrios cerrados, los cuales son destinados a los grupos sociales de mayor poder adquisitivo de la ciudad; vale remarcar que, a medida que los mismos han evolucionado,

1 Según lo establecido en su Carta Magna, el nombre oficial del municipio es "Municipio de la Ciudad de Córdoba". En este artículo se referirá a él como la "Ciudad de Córdoba", con la intención de facilitar la lectura. De esta forma, se aclara que, cuando así fuese mencionado, se alude a un territorio político-administrativo que difiere de la Provincia de Córdoba (de la cual la Ciudad de Córdoba es capital) o del Área Metropolitana de Córdoba (compuesta por el municipio en cuestión y otros aledańos). 
han incorporado sectores medios-altos. ${ }^{2}$ Es relevante recalcar que bajo las políticas habitacionales del gobierno provincial también se han desarrollado barrios que podrían ser categorizados como "cerrados". ${ }^{3}$ Por su parte, los demás actores tienden a producir vivienda para los sectores medios y bajos.

La proliferación de barrios cerrados es una de las características más significativas que explican las formas recientes del crecimiento del espacio urbano en la capital provincial. En el año 1991 comenzaron a construirse en Córdoba los primeros barrios cerrados de la ciudad, dando inicio a un proceso de fragmentación en el uso de suelo residencial. Este proceso fue facilitado por la aprobación de la Ordenanza Municipal No 8606 (Municipalidad de la Ciudad de Córdoba, 1991), que buscaba atender las demandas de las empresas de desarrollo urbano, en el marco de los nuevos acuerdos público-privados. Esta norma -destinada al desarrollo de un producto inmobiliario específico- presentaba serias contradicciones con los objetivos del marco normativo del cual era parte (Lemma, 2020b). El mismo había sido sancionado a mediados de los ańos ochenta con el objetivo explícito de frenar la expansión de la ciudad y ordenar su crecimiento. La nueva ordenanza desencadenó la proliferación de un modelo de desarrollo inmobiliario privado en forma de fragmentos residenciales, creando propuestas incluso por fuera de la legalidad que la norma planteaba.

Cabe aclarar que la proliferación de barrios cerrados es un proceso que se puede identificar en la mayor parte de las aglomeraciones latinoamericanas, siendo en las grandes metrópolis donde este proceso se evidencia con mayor claridad a partir de mediados de la década de los setenta. Los estudios urbanos han buscado explicar las características y causantes del proceso desde diferentes perspectivas, como los efectos de valorización del suelo (Abramo, 2012; Baer, 2011), la autosegregación social (Caldeira, 2007; Torres, 1998), la geografía urbana (Borsdorf, 2003), la historia (Ballent, 1998) o la antropología (Svampa, 2001).

Desde un principio, el estudio de los barrios cerrados en Argentina ha puesto especial atención en el Área Metropolitana de Buenos Aires, donde se encuentra la capital del país pero que, a su vez, es el área urbana más extensa y, por gran diferencia, la más poblada. No obstante, es posible observar una serie de estudios que ponen la lupa sobre otras ciudades latinoamericanas de escala grande e intermedia. Entre ellos, se destacan los estudios de Borsdorf, Sánchez y Marchant (2008), Frediani (2008), Lemma (2020b), Maya (2017), Pallud (2010), Prieto (2012) y Roitman (2005). El estudio de ciudades de esta escala tiende a centrarse en las

2 En este artículo se utiliza la expresión "barrios cerrados" para referirse a una serie de proyectos urbano-arquitectónicos con control de accesos, cercos perimetrales y espacios abiertos de uso exclusivo. En Latinoamérica, los diferentes países se refieren a ellos con diferentes nombres: "condominios" en Chile, "fraccionamientos cerrados" en México o "condomínios fechados" en Brasil. Según el país y ciudad en cuestión, las características y tipologías de estos varían en características formales, localización dentro de la estructura urbana, densidad habitacional, etcétera.

3 Fue posible identificar siete complejos de vivienda colectiva de desarrollo estatal construida bajo la modalidad de barrios cerrados. Solo dos de ellos (el Complejo Fuerza Aérea Alta Córdoba y Torres del Botánico) pertenecen al recorte temporal de este artículo. 
explicaciones sobre la ciudad latinoamericana que fueron hechas tomando como casos únicamente a ciudades de escala metropolitana. En este contexto, el estudio del proceso de la fragmentación residencial de la Ciudad de Córdoba busca incorporar conocimiento que permita debatir dichas explicaciones. En particular, este artículo pone el foco sobre las variables morfológicas y el tejido residencial.

Respecto de las estrategias de localización de las empresas de desarrollo urbano para sus barrios cerrados, Lemma (2020a) verifica que las mismas han apuntado a consolidar dos corredores específicos de la ciudad (el noroeste y el sur), que ya eran donde residía buena parte de la población de altos ingresos. A la par, las intervenciones estatales de vivienda subsidiada -destinada principalmente a la erradicación de asentamientos urbanos- se han localizado sobre áreas con población de bajos recursos (Marengo, 2004; Monayar, 2018). En este sentido, el autor señala que tanto las acciones privadas como las acciones públicas en la Ciudad de Córdoba entre 1991 y 2010 refuerzan una separación de la estructura urbana donde prima una polarización entre la ciudad rica y la ciudad pobre.

Poniendo la lupa sobre las características morfológicas, es posible identificar cuatro tipologías de barrios cerrados: Urbanizaciones Residenciales Especiales (URE), Barrios Cercados, Casonas y Torres (Lemma, 2020c). En este marco, los objetivos de este artículo son estudiar comparativa y cuantitativamente las diferentes tipologías de barrios cerrados; y profundizar en el análisis de las estrategias de localización a partir de la incorporación de estas variables. En este sentido, la hipótesis del artículo afirma que la evolución del proceso de fragmentación del espacio urbano cordobés en sus primeras dos décadas muestra una paulatina densificación de las tipologías existentes, junto con la aparición de nuevas tipologías y cambios en las lógicas de localización de los barrios cerrados en la ciudad. Es posible explicar la densificación y la diversificación tipológica como respuesta a un proceso de segmentación de la oferta residencial privada por parte de las empresas de desarrollo urbano, que busca atraer a consumidores cada vez más segmentados. Mientras tanto, las lógicas de localización responden a estrategias destinadas a replicar la contigüidad socioespacial existente, la cual mejora la capacidad del mercado de introducir innovaciones habitacionales.

\section{El fragmento urbano como rasgo espacial de la ciudad contemporánea}

\section{Fragmentación urbana y globalización}

El campo de los estudios urbanos se ha inclinado a explicar las causas de las transformaciones espaciales en las ciudades latinoamericanas argumentando que la estructura espacial de la mayoría de las áreas metropolitanas de América Latina se ha modificado considerablemente desde la década de los ochenta, como resultado del impacto de la globalización (De Mattos, 2010; Marcuse, 2008).

Uno de los rasgos espaciales que caracteriza a los centros urbanos producto de la globalización es la paulatina fragmentación del espacio en áreas independientes y delimitadas, produciéndose marcadas divisiones funcionales dentro de las áreas urbanas en una escala cercana a lo sectorial (Dear, 2000; Soja, 2000). Dear (2000) señala que estas transformaciones se manifiestan a escala local en estructuras 
descentralizadas y en enclaves temáticos que se cierran en sí mismos. Más aún, el autor denota la aparición de nuevas áreas centrales de trabajo que rompen con la organización espacial concéntrica de la estructura urbana tradicional. En esta línea y como respuesta a la evidencia de fragmentación espacial y social estudiada en las metrópolis californianas durante la segunda mitad del siglo xx, a partir de los años noventa se comienza a hacer hincapié en la noción de posmodernismo en la geografía urbana y el urbanismo (Dear, 2000; Soja, 2000).

En línea con estos trabajos, Secchi (2005) hace su aporte incorporando análisis de ciudades europeas. Argumenta que la estructura de la ciudad moderna estuvo dada por la premisa de la continuidad, la articulación en la división espacial y social del trabajo, y la interacción del centro con la periferia. A diferencia de esto, señala, en la ciudad y la cultura contemporáneas europeas el fragmento se consolida como una figura que quiebra con la estructura a partir de la cual se conforma la ciudad moderna; y que el resultado es una ciudad compuesta por fragmentos, estructura que, frecuentemente, es interpretada como una dispersión caótica de cosas y personas. Secchi subraya, sin embargo, la relevancia de la producción de lecturas, reconocimientos y organizaciones de estos diversos pedazos como producto de principios novedosos, como las redes, los rompecabezas o los dominó.

Cabe remarcar que la base empírica sobre la que se construye una parte importante de la teoría que explica el impacto de la globalización en las áreas urbanas, está compuesta por un conjunto de ciudades norteamericanas y europeas. En este sentido, resulta relevante alertar sobre la especificidad que las mismas puedan tener al explicar la amplia variedad de escalas y entornos urbanos latinoamericanos.

Desde el plano local, Kozak (2008) señala que la noción de fragmentación urbana ha sido utilizada en la literatura de los estudios urbanos de las siguientes tres maneras: como un proceso de separación que brinda una manera de operar en la ciudad; como un fenómeno o estado espacial dado; o como una experiencia urbana desde la perspectiva de la experimentación o percepción de la ciudad. El autor también remarca que las dos primeras son las más frecuentemente encontradas.

En un trabajo que podría encajar en el primero de estos enfoques, De Mattos (2010, p. 96) denomina como "revolución urbana" el proceso de fragmentación, policentrismo y metropolización expandida que se inició en los años setenta y que, dos décadas más tarde, se materializa a escala global. Al hablar de la escala global, el autor incorpora en específico las principales áreas metropolitanas latinoamericanas. En este marco, señala que el crecimiento expandido fue producto de un incremento de la oferta inmobiliaria de barrios cerrados, destinados a los sectores de altos ingresos. La variedad de esta oferta incluye desde las denominadas "ciudades valladas" (como Nordelta en Buenos Aires, Piedra Roja en Santiago o Alphaville en São Paulo), hasta pequeños grupos de viviendas (Hidalgo, 2004).

Si bien diversos autores incorporan la mirada sobre los procesos latinoamericanos, sus casos de estudio no sortean la preponderancia de las ciudades metropolitanas, a partir de las cuales elaboran conclusiones que tienen a ser para la totalidad de las realidades urbanas, sin importar su escala. Esta decisión metodológica fue común en varios de los estudios urbanos latinoamericanos de la época (Baer, 2011; Borsdorf, 2003; Caldeira, 2007; Catenazzi \& Lombardo, 2003; Ciccolella, 2010; Janoschka, 
2002; Vidal-Koppmann, 2014). En este sentido, en este trabajo se adhiere a la postura de Welch Guerra (2005) frente a las explicaciones dominantes sobre la globalización. Guerra seńala que la búsqueda de explicaciones locales habilita la posibilidad de los gobiernos locales y de países no centrales en cuanto a trabajar en la transformación de procesos que son globales. Con esto no se busca refutar las premisas de la globalización, sino encontrar hechos y causas específicas que sean posibles de modificar, ya que resultan poco productivos los intentos de importación acrítica y directa de fórmulas globales respecto de abordajes a partir de factores locales.

\section{El impacto de la fragmentación residencial en el espacio urbano latinoamericano} Para entender lo que sucede en el contexto latinoamericano desde una perspectiva físico-espacial, es necesario situar el momento en cuestión dentro de un proceso de transformaciones históricas del entorno construido. La fragmentación del espacio urbano latinoamericano es un tema recurrente en los estudios urbanos (Borsdorf, 2003; Carmona \& Burgess, 2001; Catenazzi \& Lombardo, 2003; Ciccolella, 2010, 2014; De Mattos, 2010; Hidalgo, 2004; Janoschka, 2002; Marengo, 2008).

A los fines de este artículo, interesa plantear dos formas de interpretar el impacto de la fragmentación sobre la estructura de las ciudades latinoamericanas. La primera de ellas es expuesta por un grupo de geógrafos alemanes que, a principio de los años 2000, realizaron una serie de estudios en las principales metrópolis sudamericanas (Borsdorf, 2003; Janoschka, 2002; Meyer \& Bähr, 2004). En dichos estudios proponen analizar y comparar las transformaciones físico-espaciales recientes desde una mirada a los cambios históricos de las ciudades. A partir de la puesta en común de estos trabajos, se construye una categorización en cuatro "modelos" de ciudad latinoamericana (Borsdorf, 2003).

Respecto del modelo de ciudad contemporánea, Borsdorf (2003) señala que se caracteriza por la reestructuración del desarrollo urbano bajo el principio de la fragmentación, y presenta un estancamiento demográfico de las principales metrópolis, en paralelo a un crecimiento por migración de las ciudades de escala intermedia y grande. Además, señala que los barrios cerrados, los centros comerciales y los parques industriales y/o de negocios son los símbolos urbano-arquitectónicos distintivos. Según el autor, la localización de estas piezas impacta en la totalidad de la estructura urbana, permitiendo visualizar contrastes socioeconómicos bruscos en la escala urbana micro.

La segunda línea es identificada por Segura (2014), quien recupera estudios más recientes en los que aparecen nuevas formas de interpretar los cambios en la estructura urbana actual y su relación con las intervenciones del sector privado. En este sentido, el autor trae a colación las explicaciones de Ciccolella (2014), De Mattos (2010) y Duhau y Giglia (2008), quienes caracterizan dichos cambios como la intensificación de la división social del espacio urbano y de los patrones de segregación previos a la proliferación de los barrios cerrados.

Asimismo y en referencia a las ciudades chilenas, Sabatini y Cáceres (2001) e Hidalgo (2004) argumentan que la fragmentación del nuevo periodo incorpora una nueva escala en la división entre ciudad rica y ciudad pobre, en la que es posible advertir una estrategia diferenciada: por un lado, se refuerza sustancialmente el patrón 
clásico de distribución de los sectores de mayores ingresos en las áreas de la ciudad donde habitan los estratos más altos; por el otro y en menor medida, se observa la consolidación de nuevos proyectos residenciales también destinados a estratos altos, pero localizados en comunas cuya matriz social de residentes es inferior.

Las posturas enunciadas presentan cierto nivel de contraposición respecto de los análisis previos sobre la localización y caracterización cuantitativa de los barrios cerrados, que identificaban un nuevo paradigma de estructuración. En este sentido, el aporte de Lemma (2020a) sobre la localización de los barrios cerrados de la Ciudad de Córdoba permite definir un proceso de fragmentación del espacio urbano que se asimila más a la intensificación de los patrones existentes (Ciccolella, 2014; De Mattos, 2010; Duhau \& Giglia, 2008), que a la aparición de un modelo novedoso que genera segregación en una escala diferente (Borsdorf, 2003; Janoschka, 2002).

\section{Estrategias de segmentación del mercado inmobiliario residencial latinoamericano}

El mercado inmobiliario formal de las grandes ciudades latinoamericanas está altamente segmentado en términos de la capacidad de compra de los usuarios (Abramo, 2012). Esto se debe a que, según afirman Harvey y Smith (2005), las empresas de desarrollo urbano han virado desde una lógica de la posición competitiva relativa, hacia una estrategia de división espacial del consumo, lo cual les permite atraer consumidores de manera segmentada. Los autores seńalan que esta estrategia les posibilita a estas empresas enfocarse en los grupos sociales con más dinero dentro del sistema y, por lo tanto, obtener mayores beneficios.

El resultado morfológico más palpable de la segmentación promovida por el mercado es la conformación de una homogeneidad socioespacial del entorno residencial más próximo; es decir, una estructura de oferta residencial segmentada en términos socioeconómicos promueve una estructura espacial fraccionada en términos socioespaciales, y la misma cristaliza la enorme desigualdad del subcontinente respecto de la distribución de la riqueza (Abramo, 2012). Los productos residenciales son utilizados por los residentes para distinguirse social y espacialmente dentro de un esquema jerarquizado, estrategia que evidencia las divisiones de clase y la estratificación socioeconómica (Bourdieu, 1994). En este sentido, sectores de riqueza consolidada y de altos ingresos se encuentran en un proceso de búsqueda de productos residenciales cada vez más específicos y distintivos, y las empresas de desarrollo urbano se vuelcan a atender tales necesidades.

Abramo (2012) explica que la promoción de los productos inmobiliarios desde una estrategia locacional de contigüidad espacial en la escala metropolitana, es uno de los caminos utilizados por los promotores inmobiliarios para disminuir la incertidumbre de los compradores respecto de la innovación de nuevas formas habitacionales. Como resultado, la extensión espacial de la ciudad formal mediante productos novedosos en sus características urbano-arquitectónicas se da a través de la promoción de dichos productos entre los estratos superiores de la pirámide de la distribución de la renta, en sectores específicos del área metropolitana. Con relación al impacto morfológico, la sumatoria de estos emprendimientos es promotora de 
una ciudad estructuralmente difusa. Como contrapartida de este proceso de difusión en el espacio urbano, el autor identifica un proceso de compactación e intensificación del uso del suelo, en el que se identifican estrategias de innovación espacial en áreas específicas; en este caso, mediante la modalidad de barrios cerrados. Estos nuevos productos inmobiliarios suponen un crecimiento de la densidad habitacional, ya que los usuarios tienden a consumir menor cantidad de espacio al optar por viviendas de menor tamaño y de mayor costo por metro cuadrado. Esta elección supone un cambio de preferencias que sustituye el tamaño del espacio residencial por una mejor accesibilidad, mejores servicios y/o un movimiento ascendente en la jerarquía de representación socioespacial de la ciudad. Se produce una segmentación de productos inmobiliarios residenciales que están dirigidos a usuarios de un estrato de ingresos levemente menor a los anteriormente mencionados. Esta tensión dual entre estrategias de difusión y compactación ha sido denominada por Abramo (2012) como "ciudad com-fusa".

En esta línea, algunos autores señalan que el modelo de ciudad vigente latinoamericano está virando desde un modelo de ciudad europeo-mediterráneo -de formas más compactas y relaciones sociales más equitativas- hacia un patrón de ciudad anglosajón-americano -donde predomina la dispersión, la segmentación, la estructuración en "islas" y las conexiones por autopistas- (Ciccolella, 2010; De Mattos, 2010). Ciccolella (2010) ha denominado a esta transición latinoamericana como la "ciudad mestiza", apelativo que Ciccolella y Vecslir (2012) explican como "la expresión territorial del enfrentamiento entre procesos de inversión y modernización sumamente selectivos en términos territoriales, entre procesos de desconcentración y recentralización, entre globalización-neoliberalización e inclusión social” (p. 24). Según Ciccolella (2010), los atributos territoriales más notorios de este mestizaje se evidencian en la yuxtaposición de rasgos formales, que van desde los precoloniales hasta los neoliberales-posmodernos; en el incremento de las desigualdades sociales, económicas y territoriales; el aumento de la brecha de direccionamiento en inversiones y un mayor proceso de hibridación cultural.

Desde una perspectiva histórica, Janoschka (2002) agrega que la transformación de una sociedad industrial a una de servicios generó una diversificación importante, en la que ambos sectores comenzaron a ofrecer a los consumidores productos cada vez más diversos para cubrir nichos de mercado cada vez más específicos. Este cambio, que denota la crisis del sistema económico fordista, dio lugar a nuevas visiones y transformaciones sobre el espacio urbano.

Las nuevas tipologías aparentan traer aparejado un aumento de la densidad residencial y, en algunos casos, de la predial (es decir, la cantidad de lotes ocupados en un área urbana). Los nuevos inmuebles ofertados se adaptan a la demanda potencial de un estrato inferior de renta, sin necesariamente reducir los precios del suelo (Abramo, 2012). Como consecuencia, el mercado reduce el tamaño medio de las residencias (respecto del stock existente), produciendo un crecimiento de la densidad habitacional que se replica en el conjunto del espacio urbano. Se concluye que el proceso de densificación del suelo en la ciudad formal sucede como respuesta a un efecto cascada en la dinámica de sustitución del espacio residencial amplio, por productos innovadores que priorizan la accesibilidad, manteniendo los servicios comunes (Abramo, 2012). 


\section{Consideraciones metodológicas}

La metodología que sirve de base a este artículo es el análisis de documentos visuales a partir de herramientas de la morfología urbana (como el trabajo de campo, observación y reelaboración de cartografías a diferentes escalas como insumo de la investigación). Principalmente fueron examinadas fotografías satelitales aportadas por la Dirección de Catastro de la Municipalidad de Córdoba, Google Earth Pro, Wikimapia y OpenStreetMap. De manera complementaria, se revisaron documentos de planificación y publicaciones de empresas desarrolladoras.

El recorte temporal efectuado comienza en el año 1991, por ser la fecha en que se sanciona la normativa que habilita la construcción de barrios cerrados y en que se construye el primero de ellos. A partir de esa fecha se toman dos periodos equivalentes de una década: 2001 marca la introducción de la tipología de Casonas; y en 2010, como resultado de la implementación de una normativa previa de convenios urbanísticos, cambia la dinámica y morfología de barrios cerrados de la ciudad. Así mismo, estas fechas permiten diferenciar un sesgo entre el desarrollo de las tipologías en el tiempo. Por último, se trata de periodos de igual duración que, además, coinciden con los censos nacionales de población y vivienda, lo cual posibilita análisis contextuales.

El recorte espacial utilizado es el de los límites de la Ciudad de Córdoba, que tiene una forma cuadrada de $24 \mathrm{~km}$ de lado y, por lo tanto, una superficie de 576 $\mathrm{km}^{2}$. Dentro del ejido municipal es posible encontrar tanto áreas urbanizadas como otras destinadas a la producción industrial y de agrocultivos. El territorio se asienta sobre la falda de una cadena montañosa denominada Sierras Chicas y es relativamente llano. Durante las dos décadas de análisis, la población de la ciudad se mantuvo estable entre los 1,1 y 1,3 millones de habitantes. A menudo en el texto se hace referencia a la Av. Circunvalación como un importante punto de referencia; la misma conforma un anillo que organiza la estructura vial de la ciudad, y es utilizada desde la normativa como límite para separar usos y formas de ocupación.

Para la clasificación tipológica se toma como base la definición desde una mirada morfológica y las categorías elaboradas por Lemma (2020c), tipologías en que se distingue entre Urbanizaciones Residenciales Especiales (URE), Barrios Cercados, Casonas y Torres (Figura 1).

FIGURA I | Fotografías representativas de tipologías de barrios cerrados en la Ciudad de Córdoba (1991-2010)

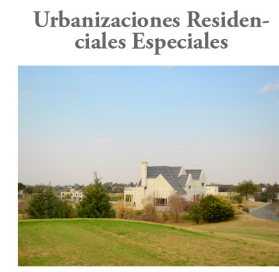

Lomas de los Carolinos

FUENTE: LEMMA (2OOOB)
Barrios Cercados

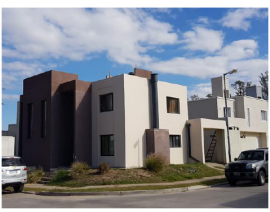

Claros del Bosque
Casonas

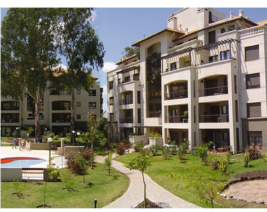

Casonas del Sur
Torres

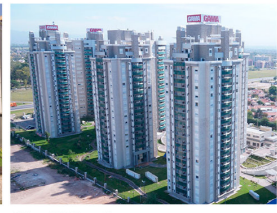

Alto Villasol 
El trabajo continúa una metodología de trabajo sobre fragmentación residencial de ciudades latinoamericanas aportada por Hidalgo (2004). Al igual que dicho trabajo, este artículo aporta conocimientos sobre barrios cerrados a partir de la indagación de variables objetivas basadas en datos empíricos y georreferenciados. Este análisis habilita explicaciones sobre la magnitud real de los fenómenos en relación con el proceso de crecimiento de la ciudad. La Figura 7 y la Figura 8 toman como referencia el esquema de representación realizado por Hidalgo para el caso de Santiago y producen piezas gráficas que habilitan una comparación con la Ciudad de Córdoba.

\section{Dos décadas de barrios cerrados en la Ciudad de Córdoba}

\section{Introducción de los barrios cerrados}

A continuación, se introducen brevemente algunas de las características que distinguen cada tipología, a los fines de comprender el análisis que sigue.

En 1991, la Ordenanza Municipal N 8608 (Municipalidad de la Ciudad de Córdoba, 1991) habilita la construcción de los primeros barrios cerrados de la Ciudad de Córdoba bajo la forma de URE; vale aclarar que esta es la única tipología que cuenta con una normativa específica que la regula. Las URE están conformadas por loteos de viviendas unifamiliares y poseen un mínimo de $30 \%$ de su superficie destinada a usos comunes (como áreas deportivas y sociales, espacios abiertos de uso exclusivo, etcétera). En promedio, tienen 186 viviendas y una superficie de predio de 48,3 ha (con valores que van desde las 5,7 ha a las 147 ha). Están destinadas a grupos socioeconómicos de alto poder adquisitivo.

Los Barrios Cercados se caracterizan por ser una serie de barrios aprobados bajo la normativa urbana vigente para urbanizaciones abiertas, pero que -en contravención con dicha norma- poseen cercos perimetrales y controles de acceso. También están conformados por loteos unifamiliares y su superficie destinada a usos comunes es mínimamente el 10\% del total. En promedio, tienen 93 viviendas y una superficie de predio de 7,8 ha (con valores entre las 0,13 ha y las 46,1 ha). Están destinados a un grupo social con alto poder adquisitivo, pero menor que el público de las URE.

Las Casonas son, inicialmente, un producto inmobiliario impulsado en 2001 por el Grupo EDIsur (uno de los principales desarrolladores urbanos de la ciudad) y luego fue adoptado por otras empresas. Normativamente, responde a diversos requerimientos zonales. Las Casonas están conformadas por viviendas agrupadas en altura de hasta cinco plantas y poseen áreas comunes muy variadas, de acuerdo con el público al que son destinadas. En promedio, tienen 80 viviendas y una superficie de predio de 0,998 ha (con valores entre las 0,24 ha y las 2,32 ha). Están destinadas a grupos sociales variados, siempre de altos y medio-altos ingresos.

Las Torres son muy similares a las Casonas y su diferencia principal es la altura: poseen seis o más plantas. En promedio, tienen 332 viviendas y una superficie de predio de 1,4 ha (con valores entre las 0,19 ha y las 4,09 ha). Están destinadas a grupos sociales variados, entre altos ingresos e ingresos medios.

Desde una perspectiva temporal, el estudio de la evolución de los barrios cerrados en Córdoba permite distinguir dos periodos marcados y tendencias hacia la segmentación de la oferta residencial fragmentada en la ciudad. En el primero se 
da la regularización de barrios privados mediante la sanción de la normativa para las Urbanizaciones Residenciales Especiales, de 1991. Estos barrios, que en su mayoría representaban un anexo de tierra rural a la trama urbana, se localizan en los sectores de la ciudad donde se alojaba la población de mayores ingresos (corredor noroeste y corredor sur) y vinculados a las vías principales de comunicación entre el centro y la periferia.

Dentro del mismo periodo y en un segundo momento, el éxito comercial de estos emprendimientos dio lugar a la aparición de los Barrios Cercados, de características similares pero que apuntaban a una mayor segmentación de la demanda, incorporando sectores de clase media y clase media-alta, y que se resuelven fuera de los marcos legales en lo que respecta a la colocación de cerramientos perimetrales.

En un segundo periodo se da una diversificación tipológica de los barrios cerrados que, en Córdoba, constó principalmente de la introducción de la modalidad de agrupación colectiva en viviendas multifamiliares, primero con las Torres e, inmediatamente después, con las Casonas.

\section{Estrategias de implantación y de generación de bordes}

La Figura 2 muestra la evolución de la ocupación de los barrios cerrados dentro del ejido municipal entre los dos periodos analizados. En primer lugar, se observa un aumento en la cantidad de barrios cerrados entre ambos. Los nuevos barrios cerrados toman como base para su ubicación las zonas del primer periodo y se extienden sobre una zona más amplia. No obstante, a diferencia de los corredores que se delinean durante el primer periodo, en el segundo se observa cierta dispersión de los barrios cerrados en dos claros sectores (noroeste y sursuroeste), con algunas excepciones, como las que presentan las Torres más céntricas y la URE Siete Soles hacia el oeste, sobre la Ruta Nacional 20, que conecta con Villa Carlos Paz.

La conformación de estos sectores evidencia no solo las áreas preferenciales de desarrollo de barrios cerrados, sino también importantes sectores donde no se desarrolla ningún tipo de estos emprendimientos residenciales. Hacia el oeste, una importante restricción la imparte la Reserva Natural de la Defensa La Calera, perteneciente al Ejército, y también la Escuela de Aviación Militar y la Fábrica Argentina de Aviones (fadeA). No obstante, en el arco comprendido entre el corredor norte, en sentido de las agujas del reloj hacia el corredor sureste, no existieron estas restricciones y, pese a ello, tampoco hay barrios cerrados. Estos grandes sectores de la ciudad fueron receptores durante ambos periodos de intervenciones residenciales estatales, que buscaban relocalizar los asentamientos informales de la ciudad (Falú \& Marengo, 2004; Monayar, 2018).

Otra transformación para destacar es la aparición y consolidación de nuevas tipologías, como las Casonas y las Torres, las cuales se muestran más propicias a ubicarse en áreas de la ciudad con mayor grado de consolidación, servicios e infraestructura urbana.

La Figura 2 también permite comparar la localización de las tipologías. Los Barrios Cercados, por ejemplo, ocupan sectores similares a los de las URE, pero más extensos en todas las direcciones. Sin embargo, durante el primer periodo, en la 
zona sur solo hubo URE; el resto de las tipologías aparece en esta zona recién durante el segundo periodo.

El incremento en cantidad, tipologías y locaciones de los barrios cerrados muestra el éxito de la modalidad residencial destinada al público de mayores ingresos de la sociedad. En este sentido, se consolida una estructura urbana conformada a partir de fragmentos.

FIGURA 2 Localización de los barrios cerrados por tipología y por periodo en la Ciudad de Córdoba (1991-2010)

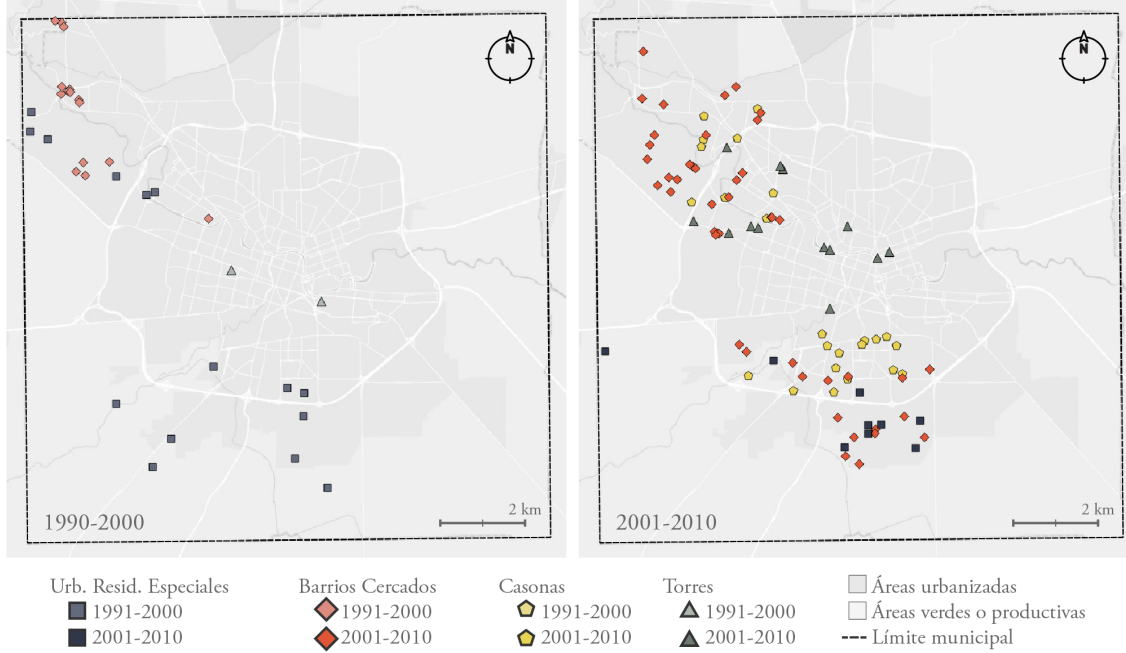

FUENTE: ELABORACIÓN PROPIA

En la Figura 3 se muestran las superficies ocupadas por barrios cerrados ordenadas según tipología y periodo. La misma permite hacer observaciones sobre las dimensiones y las cantidades de barrios construidos en cada grupo. En primer lugar, se observa que -salvo los Barrios Cercados- las tres tipologías restantes presentan una cantidad similar de casos, pero no de tamaño; sobre esta variable, se observa que las URE son las de mayor tamaño y que las mismas mantienen siempre dimensiones considerables. En segundo lugar, se observa que las tipologías con loteos individuales (URE y Barrios Cercados) difieren sustancialmente en superficie ocupada entre sí mismas, mientras que las colectivas (Casonas y Torres) tienen menos diferencia de tamaño entre ellas.

Las formas más irregulares de barrios cerrados las presentan los casos de mayores dimensiones, con una tendencia hacia la ortogonalidad a medida que los mismos se van reduciendo en tamaño. Para el caso de las URe y los Barrios Cercados, esto podría explicarse a partir del condicionamiento impuesto por las formas de las parcelas macro, que a menudo fueron el resultado de la unificación de dos o más parcelas. 
FIGURA 3 | Superficies ocupadas y formas de barrios cerrados, ordenadas por tipología y por periodo en la Ciudad de Córdoba (1991-2010)

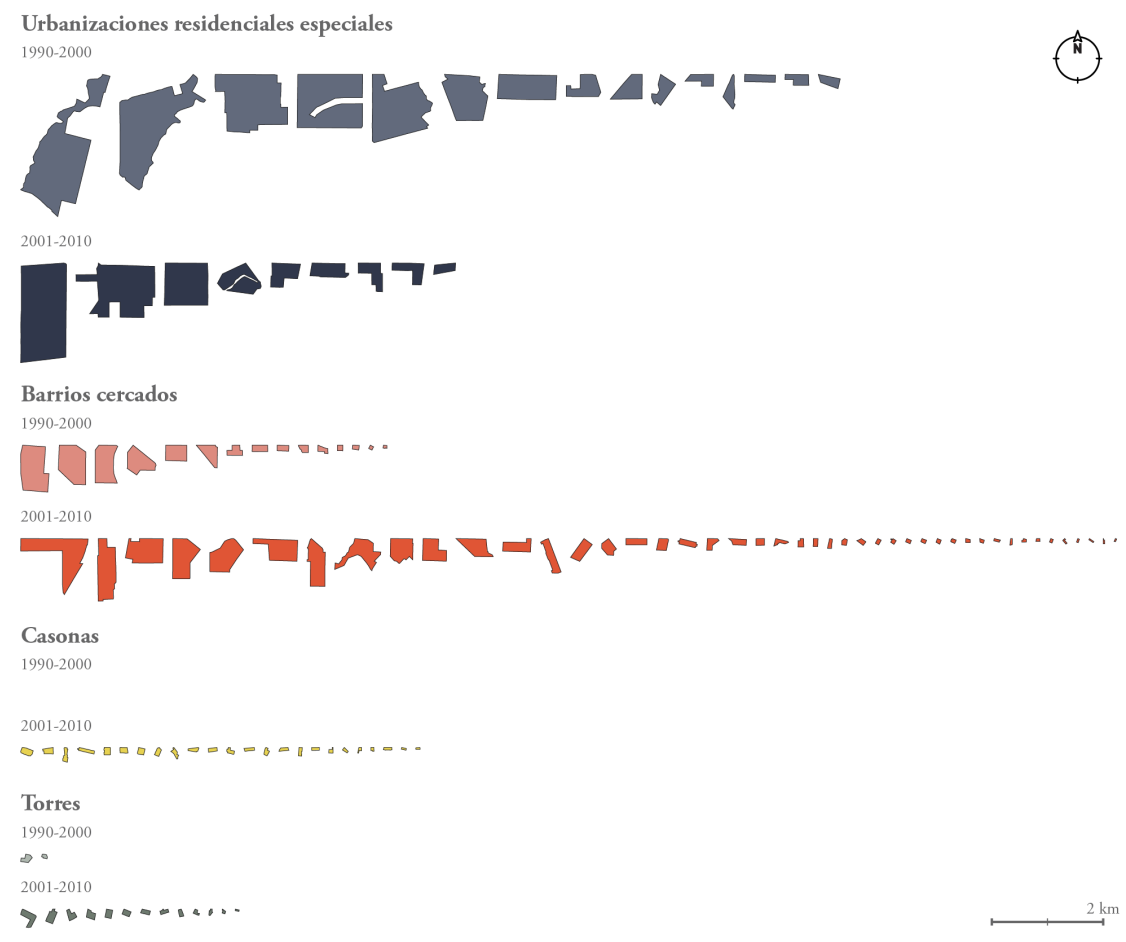

FUENTE: ELABORACIÓN PROPIA

\section{Cantidad, dimensiones y densidad}

En la Figura 4 se comparan datos agregados de las cuatro tipologías en función de los dos periodos establecidos (1991-2000 y 2001-2010). Tal como se mostró anteriormente, se puede observar un aumento sustancial en la cantidad de barrios cerrados que se construyen en el segundo periodo (91) respecto del primero (32); esto indica que, en total, en la Ciudad de Córdoba se construyeron 123 barrios cerrados entre 1991 y 2010.

Una relación similar - pero menos pronunciada- se observa en la cantidad de viviendas construidas en barrios cerrados por periodo (5.009 viviendas durante el primero y 11.936 viviendas durante el segundo).

En este sentido, el éxito de los barrios cerrados se explica principalmente por la aparición de la tipología Casonas, y de la mayor cantidad de barrios y de viviendas construidas bajo la tipología de Barrios Cercados y Torres en el segundo periodo; por el contrario, se observa que las URE es la única tipología que decrece en ambas variables. Lo anterior indica una saturación de la oferta de URE en virtud de la aparición y crecimiento de las otras tipologías.

Los datos anteriores se ven contrastados por el decrecimiento de la suma de superficie ocupada por barrios cerrados entre el primer y segundo periodo. La explicación 
de este suceso subyace en que, si bien durante el segundo periodo se construyen aproximadamente tres veces más barrios cerrados que durante el primero, las formas de agrupación de las viviendas de las tipologías del segundo periodo reflejan una menor demanda de superficie por parte de los nuevos habitantes. Este cambio se ve propiciado por la menor cantidad de URE construidas y la disminución en su tamaño promedio.

Figura 4 | Datos de barrios cerrados ordenados por periodo en la Ciudad de Córdoba (1991-2010)
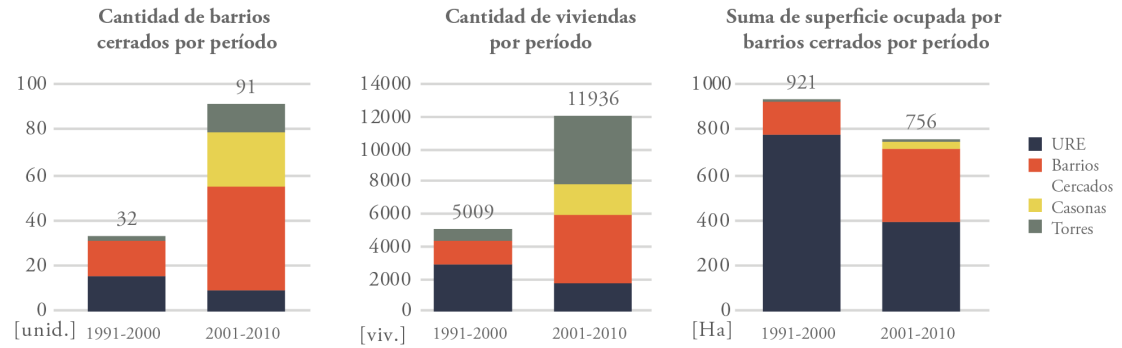

FUENTE: ELABORACIÓN PROPIA

La lectura de estos datos mediante la separación en tipologías y periodos permite observar, en la Figura 5, que Barrios Cercados fue la tipología bajo la cual se construyeron sustancialmente más cantidad de barrios cerrados, casi la mitad de ellos (60 Barrios Cercados frente a los 63 que suman las otras tipologías). Esto sucedió, en parte, por la facilidad que aporta el mecanismo de construcción de dichos barrios bajo la figura de urbanizaciones abiertas, respecto de las demás tipologías.

Por cada complejo de Torres, se construyeron cuatro Barrios Cercados. A pesar de ello, mediante la tipología de Torres se construyó casi la misma cantidad de viviendas (4.986 viviendas en Torres contra 5.585 en Barrios Cercados); esto resulta en que, si bien existen más Barrios Cercados, los complejos de Torres poseen (en promedio) 3,6 veces más viviendas. Lo anterior se debe a que los lotes de Torres tienen mayor permiso de edificabilidad.

FIGURA 5 | Datos de barrios cerrados ordenados por tipología en la Ciudad de Córdoba (1991-2010)
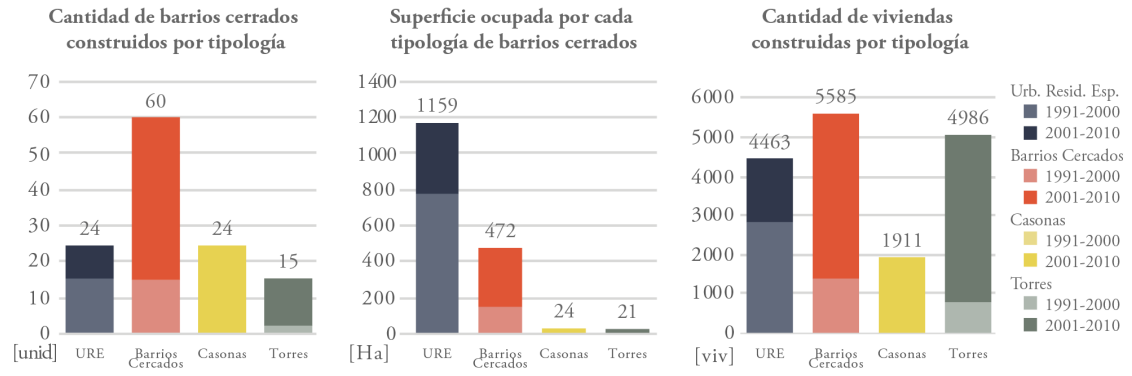

FUENTE: ELABORACIÓN PROPIA 
También se puede observar que la cantidad de barrios construidos por tipología es similar para las tipologías de URE (24), Casonas (24) y Torres (15). La relación cambia al analizar la cantidad de viviendas. Las Casonas muestran valores sustancialmente inferiores (1.911 viviendas); esto se explica, en parte, por ser un producto de un solo periodo, pero también lleva al cuestionamiento respecto de la penetración de esta tipología en el mercado.

La Figura 5 también muestra la superficie ocupada por cada tipología en el ejido municipal. En ella se denota que las URE constituyen la tipología que ocupa la mayor cantidad de superficie (1.159 ha). Tal es así, que la superficie ocupada por URE en el primer periodo es mayor que la de las otras tres tipologías sumadas para ambos periodos. Por su parte, los Barrios Cercados también presentan un valor alto de superficie ocupada (471 ha).

La diferencia sustancial en términos de superficie ocupada por cada tipología en el ejido municipal aparece entre las tipologías de agrupación unifamiliar comparadas con las multifamiliares, que son sustancialmente más eficientes en esta variable. Cabe aclarar que, si bien esta afirmación hace referencia a la totalidad de barrios construidos en cada tipología, un análisis particularizado de cada barrio, como el que se desarrolla a continuación, permite profundizar en la complejidad de esta situación.

Teniendo en cuenta que la superficie urbanizada del ejido municipal en 2010 fue 26.391 ha, es posible inferir el porcentaje de superficie que ocupan las diferentes tipologías dentro de la mancha urbana. En el caso de las URE (1.160 ha), este valor representa el 4,395\%; en el caso de los Barrios Cercados ( $472 \mathrm{ha}$ ), el 1,788\%; en el caso de las Casonas (24,0 ha), el 0,091\%; y en el caso de las Torres (21,5 ha), el 0,081\%.

Lo anterior indica que, del total de superficie destinada a la modalidad de barrios cerrados (1.677,5 ha), las uRE ocuparon el 69,15\%, los Barrios Cercados el 28,14\%, las Casonas el 1,43\% y las Torres el 1,28\%.

En la Figura 6 se muestra la variación de los valores de densidad, ordenados primero por tipología y luego en valores ascendentes. Tal como se anticipaba, las Torres son la tipología más densa de barrios cerrados. En promedio, presentan una densidad de 232 viv/ha, seguidas por las Casonas (79,8 viv/ha), los Barrios Cercados (11,8 viv/ha) y, finalmente, las URE (3,85 viv/ha).

La misma figura permite observar las siguientes cuestiones: en primer lugar, que, a medida que aumenta la densidad, las diferencias de valores dentro de una tipología son cada vez más marcadas (ver parábola de crecimiento de densidades para las Torres); en segundo lugar, que los valores de densidad de un gran número de casos son menores a las 100 viv/ha, existiendo tan solo 16 casos que superan ese valor.

La Figura 6 también permite visualizar que existen intervalos de densidad para los que diferentes tipologías pueden ofrecer respuesta. En algunas tipologías, el solapamiento de valores de densidad con otras tipologías es total. Por ejemplo, las Casonas están todas comprendidas entre las 23,2 y las 160 viv/ha.

Todos los valores de densidad de las Casonas se solapan con los de otras tipologías. En primer lugar, entre el valor mínimo de densidad de Casonas y las 86,8 viv/ha, existen Barrios Cercados que alcanzan los mismos valores. En segundo lugar, entre las 38,6 viv/ha y el máximo, hay Torres con esos valores de densidad. Este solapamiento completo de densidades de la tipología Casonas en particular, 
puede que sea otro de los motivos por los que no se construyeron tantas viviendas de esta tipología. En este sentido, visto desde la mirada de la planificación urbana, la densidad asume un valor significativo, para el que pueden aparecer diferentes respuestas tipológicas que incrementen (o no) los valores reales de ocupación.

Otro ejemplo surge de comparar los valores de densidad de las ure y los Barrios Cercados. Los casos de Barrios Cercados menos densos son similares en densidad a las URE más densas; este solapamiento sucede desde las 4,1 viv/ha hasta las 7,0 viv/ ha y corresponde a 6 Barrios Cercados y 12 URe. Cabe aclarar que, para esa última comparación, no fue tenido en cuenta el Barrio Cercado de menor densidad, por tratarse de un caso atípico. No obstante, si fuese tenido en cuenta dicho valor, se observaría que el barrio cerrado menos denso no es una URE, sino un Barrio Cercado. Esto también da cuenta de la amplia gama de respuestas morfotipológicas que pueden aparecer para un mismo valor de densidad habitacional.

\section{Figura 6 | Densidad de los barrios cerrados por tipología en la Ciudad de Córdoba (1991-2010)}

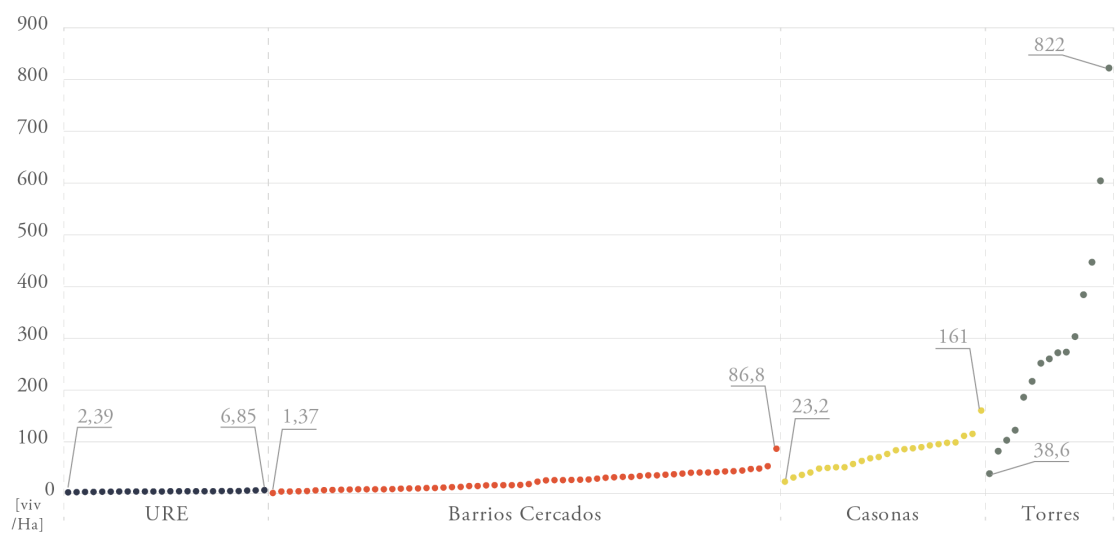

FUENTE: ELABORACIÓN PROPIA

\section{Localización y densidad habitacional}

Los gráficos presentados a continuación (Figura 7 y Figura 8) georreferencian sobre un plano de la Ciudad de Córdoba los datos levantados y construidos sobre barrios cerrados. La Figura 7 muestra la cantidad de viviendas en cada barrio con señaladores ajustados por magnitud según el dato que representan. A su vez, en las referencias se contabiliza la cantidad de casos que entran en cada categoría. Respecto de este último dato, es posible observar que la categoría con mayor cantidad de casos es la que representa los barrios con menor cantidad de viviendas; esta cantidad se va reduciendo a medida que los barrios son más grandes, incluso cuando las categorías abarcan parámetros de una mayor amplitud. Lo anterior se explica por la mayor disponibilidad de lotes pequeños (respecto de los de mayores dimensiones) y por la mayor inversión que un emprendimiento de gran escala requiere, lo cual habilita a una menor cantidad de empresas para su desarrollo. 
FIgURA 7 | Cantidad de viviendas y localización de barrios cerrados en la Ciudad de Córdoba (1991-2010)

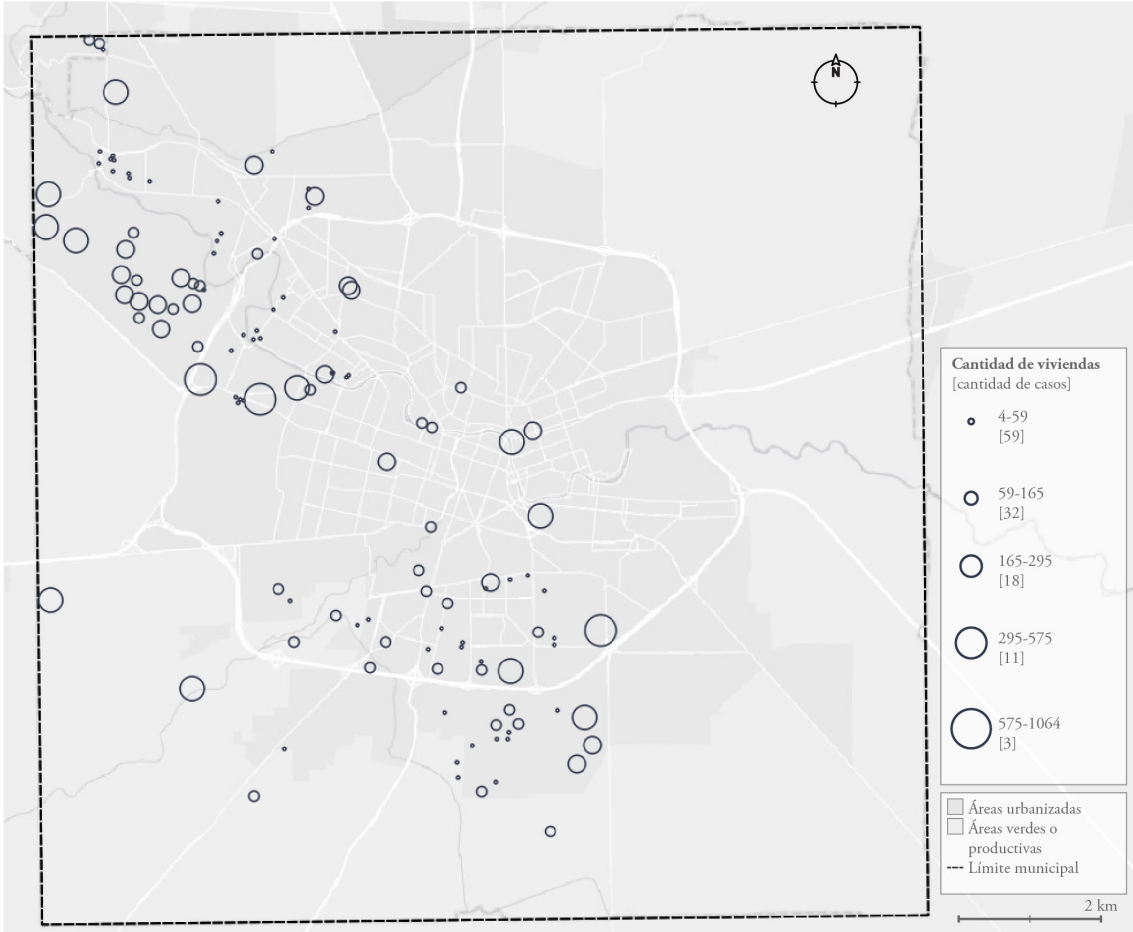

FUENTE: ELABORACIÓN PROPIA

La georreferenciación de estos datos permite distinguir diferentes áreas donde los barrios cerrados construidos presentan dimensiones similares en términos de cantidad de viviendas. Las más distinguidas son: un área que los desarrolladores denominaron Valle Escondido, compuesta por varios Barrios Cercados (sobre el corredor noroeste de la ciudad, entre la Reserva Militar y el río Suquía); al noroeste de esta área se encuentran tres de las seis URE de mayor cantidad de viviendas de la ciudad (Las Delicias, Lomas de la Carolina y El Bosque); en ese mismo corredor, marcado por la Av. Colón, pero por dentro del anillo de la Av. Circunvalación, hay también una sucesión de Torres de gran cantidad de viviendas. Los sectores donde se asientan las intervenciones de menor escala son más dispersos; el único que es posible identificar se ubica también en el corredor noroeste, próximo a la Av. Recta Martinoli. Nuevamente, esto se explica por la versatilidad que dichos lotes tienen para encajar en diferentes sectores de la ciudad. 
FIGURA 8 | Densidad bruta y localización de barrios cerrados en la Ciudad de Córdoba (1991-2010)

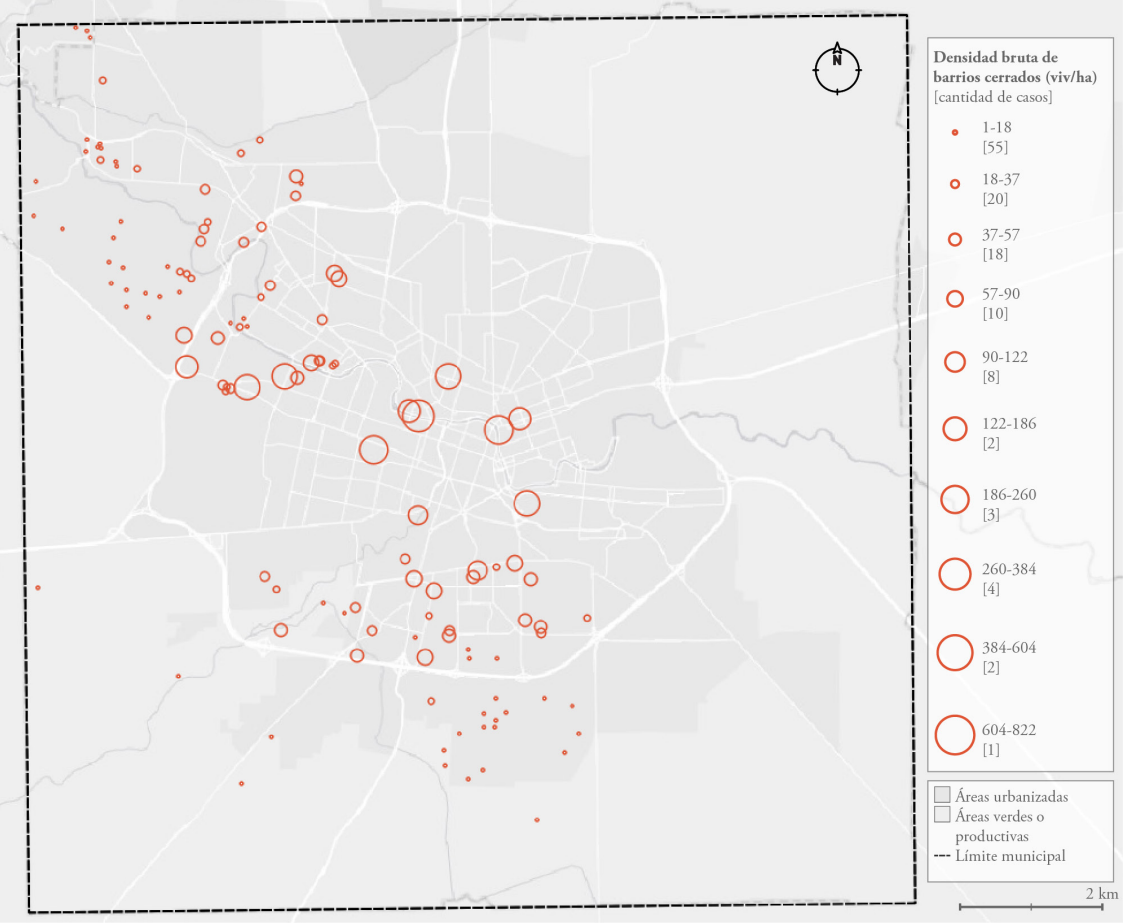

FUENTE: ELABORACIÓN PROPIA

La Figura 8 muestra la densidad residencial en cada barrio, con señaladores ajustados por magnitud según el dato que representan. Al igual que la figura anterior, en las referencias se contabiliza la cantidad de casos que entran en cada categoría. Estos datos también reflejan una tendencia decreciente de casos desde las categorías de mayor densidad hacia las de menor densidad. Esto se reproduce claramente en el gráfico, donde se observa que los barrios cerrados consolidan una tendencia hacia la conformación de una periferia muy poco densa, mientras que los casos más céntricos son unívocamente más densos. Esta situación presenta ciertas excepciones, siendo la más clara de ellas lo que sucede en el corredor de la Av. Colón, donde una serie de Torres irrumpen transversalmente, conformando un corredor de altas densidades. Ello se debe a que, en el plan de usos de suelo de la ciudad, se buscó densificar dicho corredor mediante incentivos normativos de alta edificabilidad sobre una importante disponibilidad de lotes vacantes.

A diferencia de lo que ocurría al observar el plano en función de la cantidad de viviendas (Figura 7), la siguiente figura muestra que los parámetros de densidad determinan más claramente áreas de características uniformes.

Al observar en paralelo ambos gráficos, también se deduce que en varios casos existe un correlato entre los valores de cantidad de viviendas y la densidad, 
generalmente asociados a las tipologías de Torres, aunque también de algunas Casonas, es decir, las de loteos multifamiliares. Por el contrario, se observa que las tipologías de viviendas unifamiliares a menudo presentan grandes intervenciones en términos de la cantidad de viviendas, pero las mismas son construidas con muy baja densidad, generalmente asociadas a las uRE y a los Barrios Cercados de mayor tamaño. Tal es el caso de todas las intervenciones que se localizan a continuación del corredor de Torres de la Av. Colón, pasando la Av. Circunvalación.

\section{Notas de cierre}

Entre 1990 y 2010, en la Ciudad de Córdoba se verifica la intensificación del modelo de fragmentación urbana a partir de la propagación de los barrios cerrados. Es posible llegar a esta afirmación a partir de evidencia que muestra el incremento de la cantidad de viviendas construidas bajo esta modalidad, el incremento de barrios y tipologías, y la cantidad de superficie ocupada por los mismos.

La evolución de la oferta residencial supone un quiebre entre el primer periodo (1991-2000) y el segundo (2001-2010) en términos de los modelos de organización interna utilizados en las tipologías analizadas en cada década. En otras palabras, durante el primer periodo aparecen dos tipologías de barrios cerrados de viviendas unifamiliares; mientras que, en el segundo periodo, las nuevas tipologías son de agrupamientos multifamiliares.

Desde la perspectiva que plantea Abramo (2012), la innovación surge a partir de la creación de nuevos productos residenciales cada vez más específicos y distintivos, destinados a población de mayores ingresos y que promueven una ocupación de baja densidad. En contrapartida de este proceso, la densificación surge como la apropiación de estas modalidades por parte de sectores de ingresos que tienden a consumir menos espacio. En esta línea, este proceso se verifica en el caso de la Ciudad de Córdoba, donde podría argumentarse que, posterior a las URE, aparecen las modalidades de Barrios Cercados y las Casonas como una apropiación de la modalidad fragmentada, pero esta vez con mayores densidades; posteriormente, las Torres se comercializan como un segundo paso evolutivo en la estrategia comercial que conjuga características formales y estructurantes de sus dos tipologías previas.

A la par de la aparición de nuevas tipologías de mayor densidad durante el segundo periodo, también se verifica que las tipologías existentes (URE y Barrios Cercados) disminuyen en promedio la superficie de sus intervenciones, manteniendo ciertamente estable el número de viviendas; en consecuencia, aumenta la densidad de la oferta residencial que reproduce modelos existentes, verificándose nuevamente lo que menciona Abramo (2012) respecto de las causantes del aumento de densidad residencial derivado de la diversificación tipológica. Más aún, es posible afirmar que, durante el primer periodo, se produce una saturación de la oferta en las URE, que están destinadas el segmento de más altos ingresos.

El estudio comparativo de las densidades de los diferentes casos permite inducir que, si bien cada tipología se maneja dentro de diferentes rangos de valores, estos valores se solapan con los de otras tipologías. De lo anterior se entiende que ciertos valores de densidad pueden ofrecer diferentes tipologías residenciales e incluso 
diferentes modalidades de organización de las viviendas (unifamiliares o multifamiliares). Por ejemplo, si desde la planificación se estuviese buscando garantizar 50 viv/ha, las mismas se pueden lograr tanto con un Barrio Cercado como con una Casona. En última instancia, lo anterior viene a demostrar que la adopción de ciertas tipologías no garantiza por sí misma una densidad habitacional específica, sino que otros factores proyectuales (altura de la edificación, factor de ocupación del suelo, factor de ocupación total, retiros linderos, etcétera) también estarán involucrados.

La decisión metodológica de evaluar estos datos con relación a sus formas de ocupación en el territorio que plantea Hidalgo (2004), permitió verificar en la Ciudad de Córdoba la doble tendencia hacia la consolidación de una ciudad donde es posible identificar una tensión dialéctica entre concentración y difusión del entorno construido. En este sentido, este caso de estudio cuestiona la división espacial a escala sectorial descripta por Dear (2000) y Soja (2000) para las principales áreas metropolitanas globales, encontrando dicha división en una escala urbana. Más aún, también cuestiona la falta de conformación de nuevas áreas de centralidad destinadas al trabajo, que, según los autores, sí acompañan los procesos en ciudades de escala metropolitana.

En síntesis, el estudio particularizado de ciudades latinoamericanas de escala grande e intermedia permite establecer nuevos datos que profundizan el análisis de la fragmentación. La construcción de datos y su análisis en este tipo de casos habilita la posibilidad de dar respuestas específicas a la problemática desde una mirada local. En este sentido, se adhiere a la visión de Welch Guerra (2005), quien -sin ánimo de contradecir las explicaciones generalizadoras sobre la globalización- identifica la necesidad de incorporar casos de estudio como este a las líneas de estudios urbanos.

\section{Referencias bibliográficas}

Abramo, P. (2012). La ciudad com-fusa: Mercado y producción de la estructura urbana en las grandes metropolis latinoamericanas. Revista EURE - Revista de Estudios Urbano Regionales, 38(114), 35-69. http://dx.doi.org/10.4067/S0250-71612012000200002

Baer, L. (2011). El mercado de suelo formal de la Ciudad de Buenos Aires en su contexto metropolitano. Dinámica de precios de terrenos, desarrollo inmobiliario y acceso a la vivienda en la década de dos mil. Universidad de Buenos Aires.

Ballent, A. (1998). Country life: los nuevos paraísos, su historia y sus profetas. BLOCK, (2), 88101.

Borsdorf, A. (2003). Cómo modelar el desarrollo y la dinamica de la ciudad latinoamericana. Revista EURE - Revista de Estudios Urbano Regionales, 29(86), 37-49. http://dx.doi. org/10.4067/S0250-71612003008600002

Borsdorf, A., Sánchez, R. \& Marchant, C. (2008). Aprendiendo de los errores: La necesidad de cambios a la política nacional de vivienda en ciudades intermedias chilenas. Scripta Nova, Revista Electrónica de Geografía y Ciencias Sociales, (12), 47. http://www.ub.edu/ geocrit/-xcol/166.htm

Bourdieu, P. (Dir.). (1994). La misére du monde. Seuil. 
Caldeira, T. (2007). City of Walls: crime, segregation and citizenship in São Paulo. University of California Press.

Capel, H. (2002). La morfología de las ciudades. Vol. I, sociedad, cultura y paisaje urbano. Ediciones del Serbal.

Carmona, M. \& Burgess, R. (2001). Strategic Planning \& Urban Projects: Responses to Globalization from 15 cities. Delft University Press.

Catenazzi, A. \& Lombardo, J. D. (Eds.). (2003). La cuestión urbana en los noventa en la Región Metropolitana de Buenos Aires. Universidad Nacional de General Sarmiento.

Ciccolella, P. (2010). La ciudad mestiza: metrópolis latinoamericanas atrapadas entre la globalización y la inclusión social. Revista Tamoios, 6(2), 4-16. https://www.epublicacoes.uerj.br/index.php/tamoios/article/view/1414/2999

Ciccolella, P. (2014). Metrópolis latinoamericanas: más allá de la globalización. Café de las Ciudades.

Ciccolella, P. \& Vecslir, L. (2012). Dinámicas, morfologías y singularidades en la reestructuración metropolitana de Buenos Aires. RiURB, (8), 23-41. https://core.ac.uk/download/ pdf/41789954.pdf

De Mattos, C. A. (2002). Transformación de las ciudades latinoamericanas. ¿̇mpactos de la globalización? Revista EURE - Revista de Estudios Urbano Regionales, 28(85), 5-9. http://dx.doi.org/10.4067/S0250-71612002008500001

De Mattos, C. A. (2010). Globalización y metamorfosis metropolitana en América Latina. De la ciudad a lo urbano generalizado. Revista de Geografia Norte Grande, 104(47), 81104. http://dx.doi.org/10.4067/S0718-34022010000300005

Dear, M. (2000). The postmodern urban condition. Blackwell.

Debat, M. (2016). Entre la abstracción y la realidad. Ideas y estrategias de ciudad en construcción de Córdoba y Rosario. Universitat Politècnica de Catalunya.

Díaz Terreno, F. (2011). Los territorios periurbanos de Córdoba. Entre lo genérico y lo específico. Revista Iberoamericana de Urbanismo, (5), 65-84. https://core.ac.uk/ download/pdf/41789543.pdf

Duhau, E. \& Giglia, A. (2008). Las reglas del desorden: habitar la metrópoli. Siglo xxI Editores.

Falú, A. \& Marengo, C. (2004). Las políticas urbanas: desafíos y contradicciones. En A. C. Torres Ribeiro (Comp.), El rostro urbano de América Latina (pp. 211-226). Clacso. http://biblioteca.clacso.edu.ar/clacso/gt/20100930121931/torres.pdf

Frediani, J. C. (2008). Lógicas y tendencias de la expansión residencial en áreas periurbanas de ciudades medias. Universidad Nacional de La Plata.

Harvey, D. \& Smith, N. (2005). El arte de la renta: la globalización y la mercantilización de la cultura. En D. Harvey \& N. Smith (Eds.), Capital financiero, propiedad inmobiliaria y cultura (pp. 29-58). Universitat de Barcelona.

Hidalgo, R. (2004). De los pequeños condominios a la ciudad vallada: las urbanizaciones cerradas y la nueva geografía social en Santiago de Chile (1990-2000). Revista EURE - Revista de Estudios Urbano Regionales, 30(91), 29-52. http://dx.doi.org/10.4067/ S0250-71612004009100003

Irós, G. (1991). Desarrollo urbano: reflexiones y acciones. Editorial Municipal de la Ciudad de Córdoba. 
Janoschka, M. (2002). El nuevo modelo de la ciudad latinoamericana: fragmentación y privatización. Revista EURE - Revista de Estudios Urbano Regionales, 28(85), 11-20. http://dx.doi.org/10.4067/S0250-71612002008500002

Kozak, D. (2008). Assessing urban fragmentation: the emergence of new typologies in central Buenos Aires. En M. Jenks, D. Kozak \& P. Takkanon (Eds.), World cities and urban form. Fragmented, polycentric, sustainable? (pp. 239-258). Routledge.

Lemma, M. (2017). Gestión municipal y planificación urbana en Córdoba, Argentina (19832011). Urbe. Revista Brasileira de Gestão Urbana, 9(3). https://doi.org/10.1590/21753369.009.003.AO07

Lemma, M. (2020a). Barrios cerrados: patrones de localización de tipologías y modelos de estructura urbana. Caso de estudio: Ciudad de Córdoba (Argentina) 1991-2010. Geograficando, 16(2). http://sedici.unlp.edu.ar/handle/10915/110933

Lemma, M. (2020b). Entre el modelo de ciudad compacta y las lógicas empresariales. Fragmentación del espacio urbano residencial en la Ciudad de Córdoba, 1991-2010. Universidad Nacional de General Sarmiento.

Lemma, M. (2020c). Hacia una definición y tipología de barrios cerrados. Caso de estudio: Córdoba, Argentina, 1991-2010. Estudios del Hábitat, 18(2).

Marcuse, P. (2008). Globalization and the form of cities. En M. Jenks, D. Kozak \& P. Takkanon (Eds.), World cities and urban form. Fragmented, polycentric, sustainable? (Vol. 1, pp. 25-40). Routledge.

Marengo, C. (2004). Una aproximación a la segregación residencial, como punto de partida en la formulación de políticas. Revista INVI, 19(50), 165-181. https://revistainvi.uchile. $\mathrm{cl} /$ index.php/INVI/article/view/62204

Marengo, C. (2008). Urban sprawl and spatial planning: facing the challenges of growing social inequity. Case study: Córdoba, Argentina. Tu Delft.

Marengo, C. (2013). Extensión urbana e intervenciones habitacionales. El caso de la ciudad de Córdoba (Argentina). Cuadernos de Vivienda y Urbanismo, 6(12), 280-301. https:// doi.org/10.11144/Javeriana.cvu6-12.euih

Maya, M. A. (2017). El crecimiento de urbanizaciones cerradas y la fragmentación socioterritorial en la ciudad de Mar del Plata. Pasado Abierto, 3(6), 218-240. https:// fh.mdp.edu.ar/revistas/index.php/pasadoabierto/article/view/2493

Meyer, K. \& Bähr, J. (2004). La difusión de condominios en las metrópolis latinoamericanas. El ejemplo de Santiago de Chile. Revista de Geografia Norte Grande, 32, 39-53. https:// www.redalyc.org/pdf/300/30003203.pdf

Monayar, V. (2018). Ocupación informal del espacio urbano. Situaciones, características y factores determinantes en el Municipio de Córdoba, Argentina 1990-2010. ACE, 12(36), 111-130. https://doi.org/10.5821/ace.12.36.4800

Municipalidad de la Ciudad de Córdoba. (1991). Ordenanza No 8606. Urbanizaciones Residenciales Especiales. Córdoba. https://static.cordoba.gov.ar/DigestoWeb/ pdf/87ff95ad-5f06-4113-8917-ee80465c596f/ORD_8606.pdf

Municipalidad de la Ciudad de Córdoba. (1996). Plan estratégico para la Ciudad (PEC). Córdoba.

Municipalidad de la Ciudad de Córdoba. (2001). Diagnóstico y líneas estratégicas orientativas para el Plan Urbano Ambiental. Córdoba.

Municipalidad de la Ciudad de Córdoba. (2005). Plan Estratégico de la ciudad de Córdoba. Una ciudad como su gente (PECBA). Córdoba. 
Municipalidad de la Ciudad de Córdoba. (2012). Córdoba una ciudad en cifras. Córdoba.

Pallud, P. (2010). Las urbanizaciones privadas cerradas en Santo Tomé, Santa Fe. Pampa, (6), 37-58. https://dialnet.unirioja.es/servlet/articulo?codigo=3670745

Prieto, M. B. (2012). Segregación socio-residencial en ciudades intermedias. El caso de Bahía Blanca - Argentina. Breves Contribuciones del I.E.G., (23), 129-156. https://dialnet. unirioja.es/servlet/articulo? codigo $=4322926$

Roitman, S. (2005). Who segregates whom? The analysis of a gated community in Mendoza, Argentina. Housing Studies, 20(2), 303-321. https://doi.org/10.1080/026730303042 000331790

Sabatini, F. \& Cáceres, G. (2001). Segregación residencial en las principales ciudades chilenas: Tendencias de las tres últimas décadas y posibles cursos de acción. Revista EURE Revista de Estudios Urbano Regionales, 27(82), 21-42. http://dx.doi.org/10.4067/ S0250-71612001008200002

Secchi, B. (2005). Ciudad moderna, ciudad contemporánea y sus frutos. En A. M. Ramos (Ed.), Lo urbano en 20 autores contemporáneos (pp. 145-158). Universitat Politècnica de Catalunya.

Segura, R. (2014). Desigualdades socio-espaciales en ciudades latinoamericanas. Dos problemas, una paradoja y una propuesta. Aporía Jurídica. Revista de Estudios Jurídicos y Sociales, 7(6), 11-43.

Soja, E. W. (2000). Postmetropolis: critical studies of cities and regions. Blackwell.

Svampa, M. (2001). Los que ganaron. La vida en los countries y barrios privados. Editorial Biblos.

Torres, H. A. (1998). Procesos recientes de fragmentación socioespacial en Buenos Aires; la suburbanización de las élites. En Seminario de Investigación Urbana, Instituto de Investigaciones Gino Germani, Buenos Aires. Publicado en Mundo Urbano, (3), julio 2000. https://bit.ly/3CWJFDV

Vidal-Koppmann, S. (2014). Countries y barrios cerrados. Mutaciones socio-territoriales de la región metropolitana de Buenos Aires. Dunken.

Welch Guerra, M. (Ed.). (2005). Buenos Aires a la deriva. Biblos. 